\title{
Association of hypokalemic periodic paralysis with cystic Kidney disease- A case report
}

\author{
Ricken Mehta', David Mathew Thomas' ${ }^{2}$, Vedavathi Ramakrishna ${ }^{3}$, \\ Sanjay Senaba Chikkananjaiah ${ }^{4}$, Chandrashekhar Hosadurga Rudraswamy ${ }^{5}$
}

${ }^{1}$ Senior Resident, ${ }^{3}$ Professor, ${ }^{5}$ Assistant Professor, Department of Internal Medicine, Kempegowda Institute of Medical Sciences, ${ }^{2}$ Junior Resident, Department of Internal Medicine, Bangalore Medical College and Research Institute, ${ }^{4}$ Associate Professor, Department of Radiology, Kempegowda Institute of Medical Sciences, Bengaluru, Karnataka, India

\section{A B S T R A C T}

Hypokalemic periodic paralysis (HPP) is a rare heterogenous neuromuscular disorder presenting with acute weakness. Though most cases are known to be familial or primary there are other underlying secondary causes which require evaluation and if not corrected could lead to recurrent episodes. Distal renal tubular acidosis (RTA) is one such association which has been described. Type 1/Distal RTA is known to cause renal potassium wasting thereby leading to hypokalemia. Patients with Distal RTA are unable to acidify their urine. The causes ofDistal RTAcan be hereditary, congenital, acquired or idiopathic. Severe hypokalemia with RTA has been described along with Medullary sponge Kidney and other cystic lesions in the kidney. There have been previous reports of hypokalemic paralysis occurring secondary to RTA precipitated by cystic kidney disease. We hereby report one such of hypokalemic periodic paralysis secondary to distal RTA which was associated with cystic kidney disease.

Key words: Hypokalemic paralysis, Cystic kidney disease, RTA

\section{CASE PRESENTATION}

A 52 year old man, who is a manual labourer with no known co-morbidities presented to the emergency room with complaints of two episodes of vomiting, generalized fatigue and sudden onset of weakness of both lower limbs progressing over a period of 24 hours. The pattern of weakness was such that the proximal muscles were more affected than distal. On evaluation his hemodynamic parameters were normal and cardiovascular, gastrointestinal and respiratory systems were normal. Further examination revealed that he had grade 0 power in his proximal muscles (hip and knee) while dorsi flexion and plantar flexion was possible with gravity eliminated-grade 2 power. ${ }^{1}$ There was hypotonia with absent reflexes in both lower limbs. The power in the upper limbs was normal. Bowel and bladder control was intact and there was no spinal tenderness or sensory loss noted anywhere.

About a year ago the patient had 2 similar episodes of acute weakness for which he was admitted and diagnosed with hypokalemia periodic paralysis, treated with $\mathrm{KCl}$ injections and he recovered completely. USG abdomen at that point was normal. Following this admission he had history of severe muscle cramps precipitated usually with heavy work. There was no family history of similar episodes nor did he give any history of drug intake.

His serum electrolytes at admission were - Potassium $2.0 \mathrm{mEq} / \mathrm{L}$, Sodium-140 mEq/L and Chloride- $101 \mathrm{mEq} / \mathrm{L}$. Renal function tests showed derangement with blood urea being $44 \mathrm{mg} / \mathrm{dl}$ and serum creatinine being $1.7 \mathrm{mg} / \mathrm{dl}$ which was probably due to urinary tract infection and returned to normal after a week of antibiotics. Complete hemogram showed hemoglobin of $18.8 \mathrm{~g} / \mathrm{dl}$ and a total count of 19300 cells / cu mm which was due to dehydration and urinary tract infection. The immediate priority was to correct his potassium and intravenous correction was given following which his symptoms improved in that power improved to grade 4 in both lower limbs. But serial monitoring of his potassium values done during the inpatient stay at the hospital showed low values-2.0, 2.4, 2.9, 
3.4. Serum magnesium level at admission was $1.2 \mathrm{mEq} / \mathrm{L}$ for which intravenous correction was given and after 3 days serum magnesium was $2.5 \mathrm{mEq} / \mathrm{L}$ but the serum potassium was still $2.4 \mathrm{mEq} / \mathrm{L}$.

So we worked him up for other potential causes of hypokalemia. A previous report showed a serum potassium of $3.0 \mathrm{mEq} / \mathrm{L}$ which was consistent with an assumption of chronic hypokalemia. Since he presented with only 2 episodes of vomiting probable gastrointestinal loss was not considered to be responsible for such severe hypokalemia. ABG done at the time of admission showed the following values-pH-7.38, $\mathrm{HCO}_{3}-14.4 \mathrm{mmol} / \mathrm{L}, \mathrm{p} \mathrm{CO} 2-24.6 \mathrm{mmHg}$ which was suggestive of compensated metabolic acidosis. The anion gap estimated was to be around 14.4. Serial ABG also validated similar findings. Thus it turned out that our patient had a normotensive hypokalemia with preserved anion gap metabolic acidosis. Since there are only a few causes of hypokalemia with metabolic acidosis and our patient was neither a diabetic nor on any drugs like amphotericin b or acetazolamide we were only left with RTA as the cause of hypokalemia.

A 24 hour urine examination was done which showed a total volume of $3000 \mathrm{ml}$ but the urinary potassium excretion was high- $55.2 \mathrm{mEq} / 24 \mathrm{hrs}$. The calcium excretion was normal-285mg/day. Routine urine examination was done which showed a low specific gravity of 1.008 and a $\mathrm{pH}$ of 7 which clearly showed that it was a dilute urine with a reflection of loss of acidification. It also showed WBC's and bacteria consistent with urinary tract infection. There was no evidence of glycosuria, aminoaciduria, citraturia, phosphaturia or uricosuria. A diagnosis of distal RTA was entertained as the urinary $\mathrm{pH}$ was 7-elevated in the setting systemic acidosis- $\mathrm{HCO}_{3}$ of 14.4 suggestive of RTA-mostly type 1/Distal RTA. Transtubular Potassium Gradient (T'TKG) which was calculated to be 4.2 suggestive of distal renal potassium loss. $^{2}$

Since patient had a history of alcoholism serum phosphate and calcium levels were done to rule out hypophosphatemia as a potential cause but turned out to be normal.

Parathyroid hormone levels were also done which was found to be normal.- $54.7 \mathrm{pg} / \mathrm{ml}$ to rule out hyperparathyroidism which is an important cause of Distal RTA. Sjogren's syndrome is another important cause of Distal RTA but Antinuclear antibodies were negative. Other systems were also evaluated in the form of liver function tests, 2D-Echo, Chest X-ray, Sputum examination which were unrevealing. TSH levels were also normal and thyrotoxic paralysis was also ruled out. ${ }^{3}$
USG Abdomen was done which showed prostatomegaly and multiple cysts in both kidneys which had normal size and preserved corticomedullary differentiation. Following this a CT abdomen was done which showed bilateral multiple cysts with calcifications in both kidneys with the largest measuring $2.7 \mathrm{~cm} \times 2.6 \mathrm{~cm}$ in left kidney and $3.9 \times 3.6 \mathrm{cms}$ in right kidney with a non-obstructing bilateral renal calculi. No evidence of medullary sponge kidneys. Presence of nephrolithiasis further supported Distal RTA. ${ }^{4}$

Figures 1 and 2 respectively showing multiple cysts in bilateral kidneys distributed in both the cortex and medulla with bilateral non-obstructive renal calculi.

Patient was given oral potassium and magnesium supplements and showed dramatic improvement in his symptoms and was discharged without any residual deficits proving that it was a reversible episodic paralysis caused by hypokalemia. ${ }^{5}$

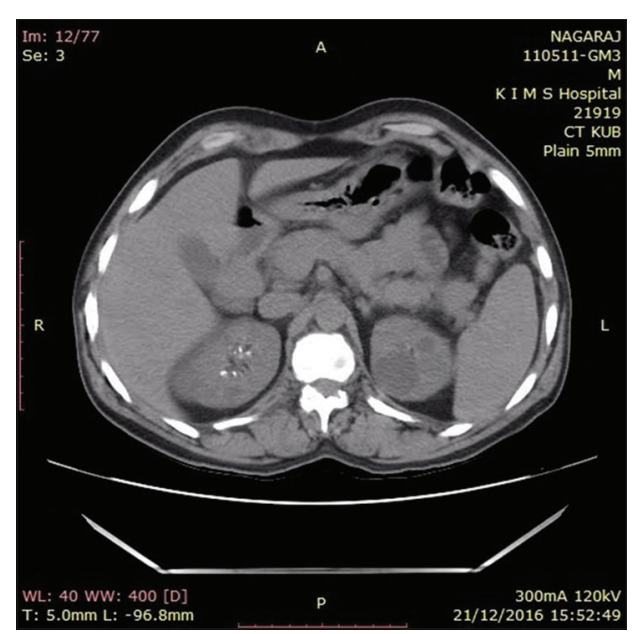

Figure 1: Bilateral Cystic Kidneys with non-obstructive calculi seen on CECT

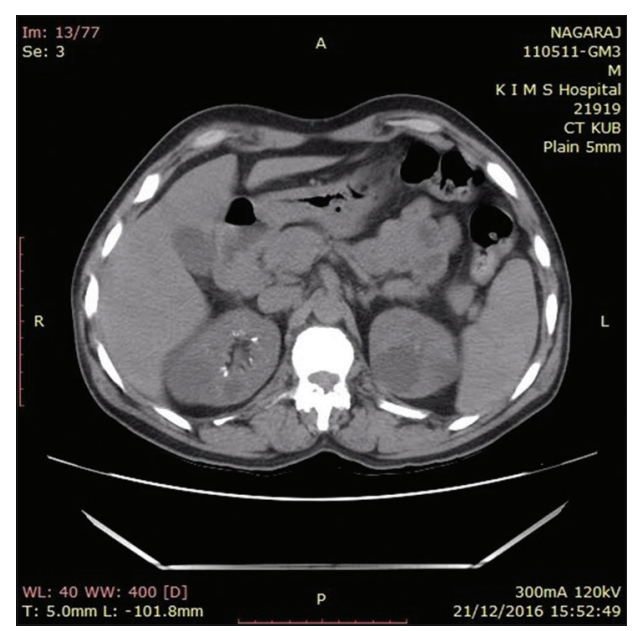

Figure 2: CECT images showing Cystic Kidneys of normal size with calculi

Asian Journal of Medical Sciences | Jul-Aug 2017 | Vol 8 | Issue 4 


\section{DISCUSSION}

Periodic paralysis are a rare group of neuromuscular disorders characterized by episodic muscular weakness caused due to fluctuations in blood potassium levels. The causes could be hypokalemia, hyperkalemia or even thyrotoxicosis. Most cases of hypokalemic periodic paralysis are channelopathies which are inherited in an autosomal dominant pattern with the exception of thyrotoxic paralysis and secondary causes of long standing hypokalemia. ${ }^{6}$ Previous studies have shown that cystic disease of the kidney is associated with hypokalemia and RTA. ${ }^{7,8}$ What causes cyst formation in kidney in the setting of hypokalemia is still not conclusive. Long standing hypokalemia is associated with a plethora of renal abnormalities which can be reversible if potassium stores are repleted. The changes include:- impaired urinary concentrating ability, intracellular acidosis, increased ammonia production, increased bicarbonate reabsorption, altered sodium reabsorption and hypokalemic nephropathy. Renal cysts arise from diverticula and segmental dilatations of renal tubules. ${ }^{9}$ Experimental studies done in rodents have shown that hypokalemia causes hyperplasia of the collecting-tubule cells in the outer medulla which is sufficient to obstruct the tubular lumen, resulting in tubular dilatation proximal to the hyperplasia and renal enlargement. Long standing hypokalemia secondary to renal loss may facilitate cyst formation by affecting proliferation of tubular epithelial cells by stimulating protein synthesis and cell division. ${ }^{10}$

The classical association of hypokalemia renal cysts and RTA is Medullary sponge kidney (MSK). Patients with MSK are often asymptomatic and often the diagnosis is incidental. RTA, nephrocalcinosis and hypokalemia are associated with MSK. MSK is diagnosed radiologically with intravenous urogram which shows cystic dilatations which have a 'bouquet of flower' appearance with contrast filling in the dilated ducts. ${ }^{4}$ Our patient did not have any features of MSK. The other possibility was that of Autosomal Dominant Polycystic Kidney Disease (ADPKD), the odd points being asymptomatic patient, normal BP, presence of normal sized kidneys on USG and CT, previous USG report of normal kidneys without any cysts done a year back, no extra-renal features suggestive of ADPKD.

\section{CONCLUSION}

Thus the conclusion we could derive was that our patient had hypokalemic periodic paralysis secondary to Idiopathic Distal RTA and the long standing hypokalemia was responsible for multiple renal cysts. Always consider Distal RTA as a possibility in such cases as long standing hypokalemia has adverse implications on the renal structure.

\section{REFERENCES}

1. Olney RK and Aminoff MJ. Weakness, myalgias, disorders of movement and imbalance. In Harrison's Principles of Internal Medicine. Volume 1. 15 edition.Edited by: Braunwald $F$, Kasper, Hauser, Longo, Jameson. New York: McGraw-Hill; 2001:118-121.

2. Lin SH, Lin YF, Chen DT, Chu P, Hsu CW and Halperin ML. Laboratory tests to determine the cause of hypokalemia and paralysis. Archives of internal medicine 2004;164(14):1561-1566.

3. Champika SSSK Gamakaranage, Rodrigo C, Jayasinghe S and Rajapakse S. Hypokalemic paralysis associated with cystic disease of the kidney: case report.BMC Nephrology 2011;12:16.

4. Caruana RJ and Buckalew VM. The syndrome of distal (type 1) renal tubularacidosis. Clinical and laboratory findings in 58 cases. Medicine (Baltimore) 1988; 67(2):84-99.

5. Ahlawat SK and Sachdev A. Hypokalaemic paralysis. Postgraduate Medical Journal 1999; 75:193-197.

6. Fontaine B. Periodic paralysis. Adv Genet 2008; 63:3.

7. Jayasinghe KS, Mendis $\mathrm{BL}$, Mohideen R, Ekanayake R, Sheriff $\mathrm{MH}$ and Dharmadasa K. Medullary sponge kidney presenting with hypokalaemic paralysis. Postgrad Med J 1984; 60(702):303-304.

8. Rao N, John M, Thomas N, Rajaratnam S and Seshadri MS. Aetiological, clinical and metabolic profile of hypokalaemic periodic paralysis in adults: A single-centre experience. The National Medical Journal of India 2006; 19(5):246-249.

9. Baert L. Hereditary polycystic kidney disease (adult form): a microdissection study of two cases at an early stage of the disease. Kidney Int 1978; 13:519-525.

10. Vicente E. Torres, William F. Young, Kenneth P. Offord and Robert R. Hattery. Association of Hypokalemia, Aldosteronism, and Renal Cysts. N Engl J Med 1990; 322:345-351.

\footnotetext{
Authors Contribution:

RM- Concept and design of the Case report, reviewed the literature, manuscript preparation and critical revision of the manuscript; DMT- Concept, collected data and review of literature and helped in preparing first draft of manuscript; VR- Conceptualized study, literature search, statistically analyzed and interpreted, prepared first draft of manuscript and critical revision of the manuscript; SCC-Concept of study, and Interpretation of collected data; $\mathbf{C H}$ - Concept and Review of Case Report.

Orcid ID:

Dr. Ricken Mehta:D http:// orcid.org/0000-0001-7825-405X

Dr. David Mathew: http://orcid.org/0000-0003-2325-1614

Dr. Vedavathi Ramakrishna:@ http:// orcid.org/0000-0002-9870-7404

Dr. Sanjay Chikkananjaiah: http:// orcid.org/0000-0001-5377-0939

Dr. Chandrashekhar Rudraswamy: http:// orcid.org/0000-0001-9449-0394

Source of Support: Nil. Conflict of interest: None declared.
} 